\title{
A powerful tool for genome analysis in maize: development and evaluation of the high density 600 k SNP genotyping array
}

\author{
Sandra Unterseer ${ }^{1}$, Eva Bauer ${ }^{1 *}$, Georg Haberer $^{2}$, Michael Seidel $^{2}$, Carsten Knaak ${ }^{3}$, Milena Ouzunova ${ }^{3}$, \\ Thomas Meitinger ${ }^{4}$, Tim M Strom ${ }^{4}$, Ruedi Fries ${ }^{5}$, Hubert Pausch ${ }^{5}$, Christofer Bertani ${ }^{6}$, Alessandro Davassi ${ }^{6}$, \\ Klaus FX Mayer ${ }^{2}$ and Chris-Carolin Schön ${ }^{1 *}$
}

\begin{abstract}
Background: High density genotyping data are indispensable for genomic analyses of complex traits in animal and crop species. Maize is one of the most important crop plants worldwide, however a high density SNP genotyping array for analysis of its large and highly dynamic genome was not available so far.

Results: We developed a high density maize SNP array composed of 616,201 variants (SNPs and small indels). Initially, $57 \mathrm{M}$ variants were discovered by sequencing 30 representative temperate maize lines and then stringently filtered for sequence quality scores and predicted conversion performance on the array resulting in the selection of 1.2 M polymorphic variants assayed on two screening arrays. To identify high-confidence variants, 285 DNA samples from a broad genetic diversity panel of worldwide maize lines including the samples used for sequencing, important founder lines for European maize breeding, hybrids, and proprietary samples with European, US, semi-tropical, and tropical origin were used for experimental validation. We selected $616 \mathrm{k}$ variants according to their performance during validation, support of genotype calls through sequencing data, and physical distribution for further analysis and for the design of the commercially available Affymetrix ${ }^{\circledR}$ Axiom ${ }^{\circledast}$ Maize Genotyping Array. This array is composed of 609,442 SNPs and 6,759 indels. Among these are 116,224 variants in coding regions and 45,655 SNPs of the Illumina ${ }^{\oplus}$ MaizeSNP50 BeadChip for study comparison. In a subset of 45,974 variants, apart from the target SNP additional off-target variants are detected, which show only a minor bias towards intermediate allele frequencies. We performed principal coordinate and admixture analyses to determine the ability of the array to detect and resolve population structure and investigated the extent of LD within a worldwide validation panel.

Conclusions: The high density Affymetrix ${ }^{\circledast}$ Axiom ${ }^{\oplus}$ Maize Genotyping Array is optimized for European and American temperate maize and was developed based on a diverse sample panel by applying stringent quality filter criteria to ensure its suitability for a broad range of applications. With $600 \mathrm{k}$ variants it is the largest currently publically available genotyping array in crop species.
\end{abstract}

Keywords: High density genotyping array, Maize, SNP

\footnotetext{
* Correspondence: e.bauer@tum.de; chris.schoen@tum.de

${ }^{1}$ Plant Breeding, Centre of Life and Food Sciences Weihenstephan,

Technische Universität München, 85354 Freising, Germany

Full list of author information is available at the end of the article
} 


\section{Background}

High-throughput genotyping has revolutionized genetic analyses in humans, livestock species, crop and model plants in the past decade [1-3]. Covering genomes with high resolution, single nucleotide polymorphism (SNP) genotyping arrays facilitate the detection of associations between SNPs and phenotypes. They represent a powerful tool for dissecting complex traits via genome-wide association studies (GWAS) or quantitative trait locus (QTL) analysis as well as for fine mapping genes of interest and forward genetics cloning strategies [4-7]. In addition, they are broadly used in crop and livestock breeding for germplasm characterization and marker assisted selection [8]. The availability of high density genotyping arrays has enabled breakthroughs in genome-wide approaches such as genomic prediction and detection of selection signatures [9-12]. Here, we describe the development of the currently largest publicly available SNP array in crop species and discuss its potential for different applications in maize.

Maize is one of the most important crops worldwide serving as food, livestock feed, and component of industrial products. A key step in corn production was the establishment of divergent heterotic patterns for hybrid breeding [13]. Most worldwide hybrid breeding programs exploit heterotic effects between different subgroups within the Dent pool, whereas crosses between the two maize pools, Dent and Flint, are mainly used in hybrid breeding for the cooler regions in Central Europe. Maize production has continuously risen over time, but to further increase selection gain and accelerate breeding processes profound knowledge is required regarding genes and genomic regions involved in agronomically important traits.

Genotyping arrays offer an efficient alternative to whole genome sequence data for gaining genomic information in high-throughput. However, the establishment of a high density genotyping array requires the identification of a large number of variants polymorphic in a representative discovery panel to ensure its utility for a wide range of approaches and study designs. In maize, the identification of sequence variants for genomic analyses faces specific challenges due to its evolutionary history and high variability of its genome. As an ancient polyploid species, the maize genome is characterized by numerous duplicated chromosomal regions giving rise to paralogous sequences [14-16]. A reference sequence exists for maize, which covers around $90 \%$ of the $2.4 \mathrm{~Gb}$ genome of inbred line B73 (AGP_v2), but the high amount of transposable elements, paralogs, copy number variants $(\mathrm{CNV})$ as well as structural variants like presence/absence variants (PAV), is a challenge for reliable sequence read alignment and variant identification due to ambiguous sequence read mapping results $[15,17,18]$. Despite recent reports like the comprehensive genotyping of the USA national maize inbred seed bank [19] using SNPs identified through genotyping by sequencing (GBS) at low sequence coverage [20], sequencing-based approaches such as GBS have to cope with large amounts of missing data and require the establishment of demanding bioinformatics pipelines and imputing algorithms, which may not be routine in all labs.

The highest resolution of a commercially available genotyping array for maize has been achieved by the Illumina ${ }^{\circ}$ MaizeSNP50 BeadChip [21]. It has been used extensively for genetic studies [22-25] and is composed of $50 \mathrm{k}$ usable SNPs. This number of SNPs is in the same range as for recently published genotyping arrays for rice [8], soybean [26], and wheat [27], but much lower compared to high density genotyping arrays which are available for animal species, e.g. chicken [28] and cattle with $648 \mathrm{k}$ and $777 \mathrm{k}$, respectively [29,30], as well as for humans with more than $900 \mathrm{k}$ SNP variants [5]. Especially for maize with its large genome size and high level of diversity, high marker resolution is desirable. In addition, linkage disequilibrium (LD) decays rapidly in some germplasm, e.g. in landraces or highly diverse sample panels [31] emphazising the requirement of higher marker densities than so far available on genotyping arrays.

We selected sequence variants for the design of a high density $600 \mathrm{k}$ SNP genotyping array for maize based on $57 \mathrm{M}$ SNPs and small indels that were discovered by mapping whole genome sequencing reads of 30 representative temperate maize lines against B73 AGP_v2. For experimental validation, we selected $1.2 \mathrm{M}$ variants by applying stringent filtering criteria. This $1.2 \mathrm{M}$ subset was used to genotype 285 maize samples representing the genetic diversity of European (EU) and American (US) temperate maize as well as a sample of tropical maize lines. We created a final selection of 616,201 high quality variants based on their assay performance, physical distribution, and concordance with in silico variant calls from sequencing data. Here, we describe the design of the high density Affymetrix ${ }^{\circ}$ Axiom ${ }^{\circ}$ Maize Genotyping Array which represents a powerful tool for fine-mapping of genomic regions, genome-wide studies, and detection of marker-trait associations. We also demonstrate its application for investigating subpopulation structure and LD in diverse maize germplasm.

\section{Results and discussion}

\section{Discovery and pre-selection of variants}

For variant (i.e. SNP and indel) discovery we sequenced 30 maize inbreds composed of 17 European Flint lines as well as nine European and four US Dent lines (Additional file 1: Table S1). The lines represent important founder lines for maize breeding in Europe and the US and have been used in previous studies 
$[32,33]$. Mapping the generated sequence reads to the B73 reference sequence (AGP_v2) resulted in 50-fold sequence coverage on average of four deep sequenced lines (DK105, EP1, F7, PH207) as well as 12-fold coverage on average of the 26 remaining lines. Based on the mapped sequence reads 56,938,462 variant positions were identified.

A filtered list of variants was created for quality score determination similar to the dual approach of Chia et al. [18]. Variants were included in this list if they were identified independently by two different programs, SAMtools [34] and GATK [35] and were characterized by high quality scores as well as presence of reference (B73) and non-reference alleles in the discovery panel. Applying these filters, the initial variant number was reduced by a factor of 10 . We finally selected 5,593,169 bi-allelic variants for further analysis. $66.7 \%(3,731,960)$ of these variant positions were congruent with variants reported by [18] for the maize HapMap2 data. Of 46,660 variants from the Illumina ${ }^{\oplus}$ MaizeSNP50 BeadChip which could be uniquely anchored to the B73 reference sequence, 43,615 (93.5\%) were also covered by in silico SNP calls from sequencing in our set of $5.6 \mathrm{M}$ variants. This proportion is higher than the $72.3 \%$ overlap reported in the maize HapMap2 SNP dataset reported by [36] and can most likely be attributed to the higher sequence coverage in our study.

\section{Selection of high-confidence variants for array construction}

A multi-step filtering approach was applied to reduce the number of $5.6 \mathrm{M}$ variants to a subset of $1.2 \mathrm{M}$ variants for experimental validation on two Affymetrix ${ }^{\odot}$ Axiom $^{\circledast} 600 \mathrm{k}$ screening arrays (Figure 1). From those, $616 \mathrm{k}$ were selected for the design of the $600 \mathrm{k}$ array.

\section{Variant selection according to in-silico analysis of sequence data}

The 5.6 M variants were filtered according to quality and their support by sequence reads. The sequenced lines were inbred lines with only minor residual heterozygosity (mean of $0.65 \%$, Additional file 1: Table S2) as determined from Illumina ${ }^{\circ}$ MaizeSNP50 data. In the 5.6 $\mathrm{M}$ variants, we observed $23.3 \%$ heterozygous compared to $72.7 \%$ homozygous calls, which was not expected from the Illumina ${ }^{\bullet}$ MaizeSNP50 genotyping data.

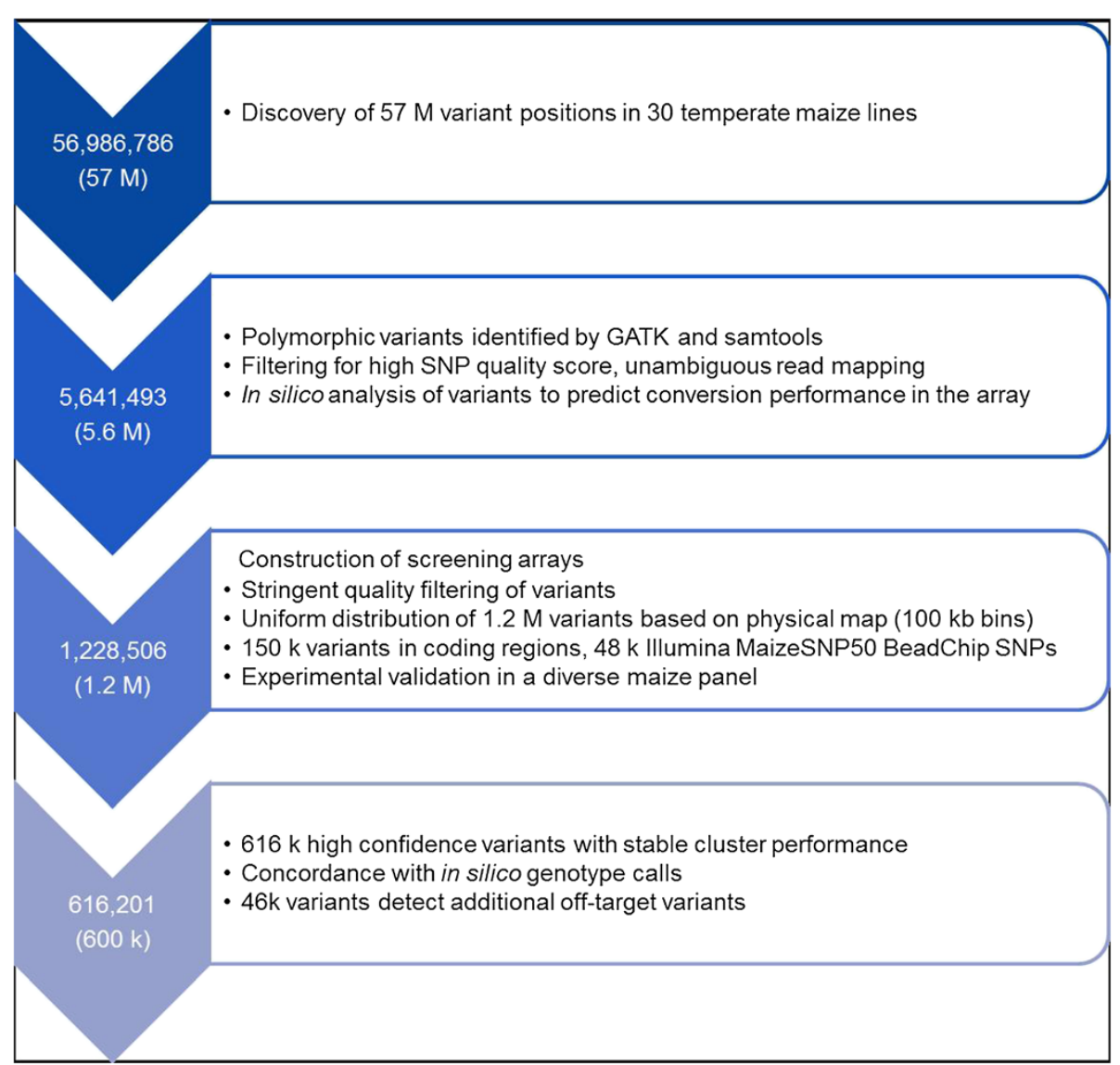

Figure 1 Flow diagram with the major filtering steps. Flow diagram showing steps and major criteria of the variant selection process during development of the maize $600 \mathrm{k}$ genotyping array. 
Besides "true" heterozygous calls, such calls may arise from the large fraction of segmental duplications as well as orthologous and paralogous sequences retained in the ancient polyploid maize genome [15]. In line with this, the false discovery rate (FDR) of heterozygous calls was significantly higher $(87.0 \%)$ compared to the FDR of homozygous calls (1.6\%) as determined by comparison with variant calls from the Illumina ${ }^{\circ}$ MaizeSNP50 BeadChip. Thus, in order to create a list of high quality variants only homozygous calls were considered for further analysis.

We decided to include all available 150,394 coding variants on the screening arrays, as these variants have a greater potential than non-coding variants to affect gene function. To enable comparison across studies, we further included 48,324 SNPs of the Illumina MaizeSNP50 BeadChip as "must-have" variants. The remaining $\sim 1 \mathrm{M}$ positions on the screening arrays were filled with noncoding variants based on their distribution across the genome. Similar to the strategy reported by Kranis et al. [28], we applied a bin based approach with the intention to create a subset of physically equally distributed variants. We observed that variant numbers in centromeric bins were always lower than in telomeric bins, indicating lower polymorphism rates in the centromeric regions. This reduction of variant numbers around the centromeres was also observed in other maize studies [18,19,37] and may result from the high proportion of repetitive DNA around the centromeres for which no markers can be developed. Aiming simultaneously for a balanced representation of pool-specific as well as shared variants between Dent and Flint, 931,340 variants were included in the list for validation. We selected 158,448 additional variants to specifically increase the number of variants in under-represented bins to reach a final number of $1,228,506$ variants which could be placed on the screening arrays. The marker density on the screening arrays was one variant per $\sim 1.7 \mathrm{~kb}$ on average over all chromosomes (Additional file 1: Table S3).

\section{Variant validation by genotyping 285 representative maize samples}

In order to assemble a robust set of variants for design of the $600 \mathrm{k}$ array, the selected set of $1.2 \mathrm{M}$ variants was used to genotype 285 DNA samples from 280 diverse worldwide maize inbred lines and hybrids for the evaluation of variant performance (Additional file 1: Table S4). We investigated conversion performance of the variants on the array with respect to (i) genotype call rates, cluster separation, and reproducibility, (ii) polymorphism in the panel under study, and (iii) consistent Mendelian inheritance from parents to off-spring in trios.

Hybridization intensity signals were clustered by the Affymetrix Axiom GT1 algorithm and interpreted as homozygous, heterozygous, or no calls, respectively. Different from the situation in humans or animals, where samples are highly heterozygous, most of the samples in our maize validation panel were highly inbred. Thus, we compared genotype calls obtained with and without applying an inbred correction factor (Additional file 2: Figure S1). This factor was assigned to each sample to adjust the probability of observing a heterozygous call given the inbreeding level of the sample. The average call rate of the screening arrays could be increased by $2.3 \%$ to $98.1 \%$ upon inbred correction (Additional file 1: Table S5). With inbred correction, inbred line B73 exhibited the highest call rate $(99.5 \%)$ and one F1 hybrid (UH007 x Lo11, 92.2\%) together with Teosinte (acc. GID265285, 92.2\%) the lowest call rates. Furthermore, American maize lines revealed higher call rates on average compared to European lines, followed by call rates of tropical lines and hybrids. This is in accordance with the literature [21] and suggests a negative correlation between call rate and increasing sequence divergence to the reference sequence of B73 from which probe sequences on the array were derived.

Based on genotype call cluster separation, cluster variance, and cluster position, variants were assigned to one out of six quality categories (Additional file 2: Figure S2). Comparing the category assignments with and without inbred correction resulted in a change of category in 36.2\% of all variants (Additional file 1: Table S6). As expected, the category of variants fulfilling all cluster metric criteria and classified as "PolyHighResolution" (PHR) increased most, resulting in a gain of $30.7 \%$ upon inbred correction. Details on the number of variants from each category with and without inbred correction are given in Additional file 1: Table S6. In total, $25.1 \%$ of the newly developed $1,131,860$ variants (excluding the Illumina ${ }^{\circ}$ MaizeSNP50 variants) failed to convert and did not give reliable genotype calls upon inbred correction (designated "other" in Additional file 1: Table S6). The proportion of $74.9 \%$ converted variants is lower than in a similar study in chicken, where $82.0 \%$ of the variants could be converted into successful variants [28]. In rice which has an around five-fold smaller and less complex genome than maize, $84 \%$ of variants of the Illumina ${ }^{\circ}$ RiceSNP50 array [8] were converted successfully (GenTrain score $>0.5$ ). Given the higher complexity of the maize genome compared to chicken or rice, our conversion rate is in the expected range.

\section{Selection of high-confidence variants and composition of the $600 k$ array}

For the selection of high-confidence variants for the $600 \mathrm{k}$ array, we applied a voting system based on (i) their performance on the screening arrays, (ii) concordance of array genotyping calls with in silico variant calls from sequencing data of the 30 maize lines in the discovery 
panel, and (iii) over- or under-representation of the corresponding bin. To ensure a high performance on the final array, the highest weight was assigned to the first criterion. We focussed on clearly separated genotype clusters with little variance that were not influenced by information regarding the inbreeding level (Additional file 2: Figure S1). Applying this procedure the 570,546 highest scoring variants as well as 45,655 SNPs of the Illumina MaizeSNP50 BeadChip were included in the final selection for the $600 \mathrm{k}$ array (Additional file 1: Table S6).

The $600 \mathrm{k}$ genotyping array is composed of 616,201 variants (609,442 SNPs and 6,759 indels), corresponding to an average density of one variant per $\sim 3.4 \mathrm{~kb}$ (median density one variant per $0.3 \mathrm{~kb}$; Additional file 1: Table S3, Additional file 2: Figure S3). The average genetic distance between variants is $0.0025 \mathrm{cM}$, which corresponds to 406 variants per cM. The variants are evenly distributed across the chromosomes with the only exception of one region on the short arm of chromosome 6 , where the maximal distance between neighboring variants exceeds 1.2 Mb. Despite a specific filter aiming for equal variant distribution according to the physical map distance, the final distribution followed the average recombination rate along chromosomes, which reflects varying polymorphism rates in the material under study (Figure 2). The highest density of variants was found in gene enriched telomeric regions, thus ensuring the maximal possible amount of genetic information in regions with high recombination rates. A comparable pattern of variant distribution as well as a lack of variants on the short arm of maize chromosome 6 in the nucleolus organizer region (NOR; approximate position 7$28 \mathrm{Mb}$ ) has been reported previously $[18,19]$. From the 616,201 variants represented on the Affymetrix ${ }^{\oplus}$ Axiom $^{\odot}$ Maize Array 561,751 (91.2\%) are also present in the maize HapMap2 variants [18].
All 616,201 variant positions were annotated based on the B73 filtered gene set which comprises 39,656 genes (Additional file 1: Table S7), resulting in 26,620 genes (67.1\%) tagged with at least one variant in their coding, intronic, or UTR region, compared to 17,520 genes tagged by SNPs of the Illumina ${ }^{\circ}$ MaizeSNP50 BeadChip (44.2\%). Including $5 \mathrm{~kb}$ up- and downstream regions, 35,089 genes $(88.5 \%)$ were represented by at least one variant, thus providing an excellent basis for finding marker-trait associations in targeted and genome-wide approaches.

To determine the reproducibility of variants represented on the $600 \mathrm{k}$ array, technical and biological replicates were analysed. First, three technical B37 replicates as internal controls exhibited up to $99.8 \%$ of identical genotype calls (Additional file 1: Table S8). Three biological replicates from different seed sources exhibited a high level of concordant genotype calls in the range of 99.76\% to 99.84\%. Furthermore, two lines (DK105 and EP1) were represented by two samples each comprised of a single plant and a pooled sample, respectively, showing $99.51 \%$ and $97.73 \%$ concordance. Some lack of concordance here can be explained by residual heterozygosity in the pooled samples. For determination of stable Mendelian inheritance, 23 trios with both parental lines as well as the corresponding F1 hybrid were analysed. These trios revealed stable Mendelian inheritance between parental lines and their offspring in $94.3 \%$ of the variants. After excluding the trio with the lowest call rate (UH007, Lo11, UH007 x Lo11) stable Mendelian inheritance could be observed in $97.6 \%$ of the variants, underlining the call rate as an indication of sample quality. The analysis of biological and technical replicates and trios confirmed the high reproducibility of genotype calls obtained with the variants represented on the Affymetrix ${ }^{\circ}$ Axiom $^{\odot}$ Maize Array which is in the same range as reported for the Illumina ${ }^{\oplus}$ MaizeSNP50 BeadChip [21].

\section{A}

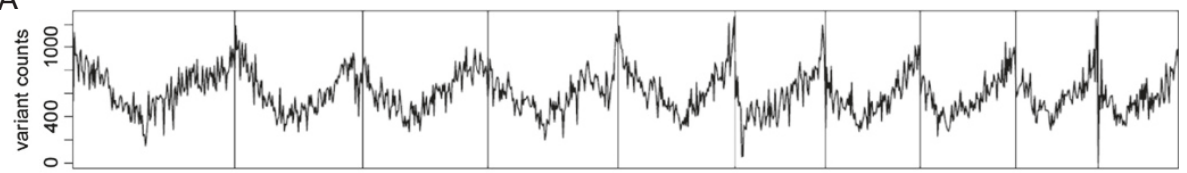

B

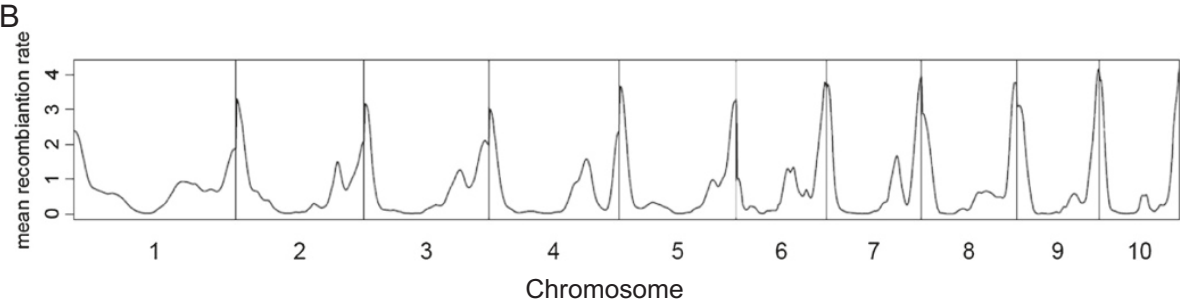

Figure 2 Physical distribution of $\mathbf{6 1 6} \mathbf{k}$ variants and recombination rate. Physical distribution of variants and average recombination rate along the ten maize chromosomes depicted for $2 \mathrm{Mb}$ windows. A) Distribution of $616 \mathrm{k}$ variants represented on the $600 \mathrm{k}$ array, B) Average recombination rate in $\mathrm{cM} / \mathrm{Mb}$ from [32]. 
The usefulness of a genotyping array is characterized by the number of variants polymorphic in the panel of genotypes under study. In the 155 public maize lines, two Teosinte accessions, and $23 \mathrm{~F} 1$ hybrids used in this study for validation, $99.9 \%$ of the $600 \mathrm{k}$ array variants were polymorphic. Only a small number of 262 variants (all derived from the Illumina ${ }^{\circ}$ MaizeSNP50 BeadChip) were monomorphic across all samples of the validation panel. After excluding three genotypic samples without clear germplasm group assignment, $95.6 \%$ of the $600 \mathrm{k}$ variants were polymorphic within Dent $(\mathrm{N}=73), 98.7 \%$ in Flint $(\mathrm{N}=79)$, and $97.2 \%$ within $\mathrm{F} 1$ hybrids $(\mathrm{N}=23)$, respectively (Figure 3). Only $42.2 \%$ of the variants were polymorphic within the two Teosinte accessions. It must be noted however, that with only two samples the diversity in Teosinte is not well captured in our validation panel. Additionally, the array was not optimized for wild maize relatives as they were not included in the discovery panel. The high overall polymorphism rate depicts the quality of the filtering procedure and is in line or even exceeding results obtained by other studies regarding genotype array validation in animals and plants $[8,21,38,39]$. It confirms the utility of the array for a wide range of applications in maize germplasm.

Among the selected variants, one category called "OffTarget Variants" (OTVs) was of special interest since these 45,974 variants detect previously uncharacterized variants in the flanking region of the target variant. Due to a reduced hybridization efficiency OTVs are characterized by cluster splits or additional relatively low signal intensity clusters compared to expected homozygous and heterozygous genotypes (Additional file 2: Figure S2) and have been shown to be reproducible [40]. These $46 \mathrm{k}$ variants offer the possibility not only to analyse the genotype call of the target variant, but provide in addition information on presence or absence of putative additional variants in the flanking regions. The latter information can be treated as a bi-allelic flanking variant and was included for population structure analyses.

\section{Analysis of population substructure}

The identification of population substructure is crucial for quantitative genetic or population genetic studies since population stratification or admixture may affect detection of marker-trait associations, genomic prediction, or estimation of population genetic parameters. To determine the ability of the variants represented on the maize $600 \mathrm{k}$ genotyping array to resolve population structure, we performed principal coordinate (PCoA) and admixture analyses of 155 public inbred lines. The first principal coordinate revealed a clear separation of Dent and Flint with a small group of samples located in the center (Figure 4A). This central group included (sub)tropical Flint and Dent lines, the popcorn accession, two lines with unknown pedigree as well as Flint lines that originated from Southern Spain and one Flint line tracing back to Argentina. The clear separation of Dent and Flint reflects their genetic differentiation for more than 2,500 years [41], accompanied by varying adaptive and selective pressures. Similar results were obtained in studies based on isozymes [42], RFLPs [43,44], SSRs [41], and SNPs [45]. Analyzing the 73 Dent lines separately (Figure 4B), the first two axes further subdivided the samples into distinct subgroups, namely Iowa Stiff Stalk Synthetic (BSSS), Iodent, and Lancaster Sure Crop (LSC), with several non-BSSS and tropical lines in the center. The three groups BSSS, non-BSSS (including LSC), and Iodent represent three major heterotic groups

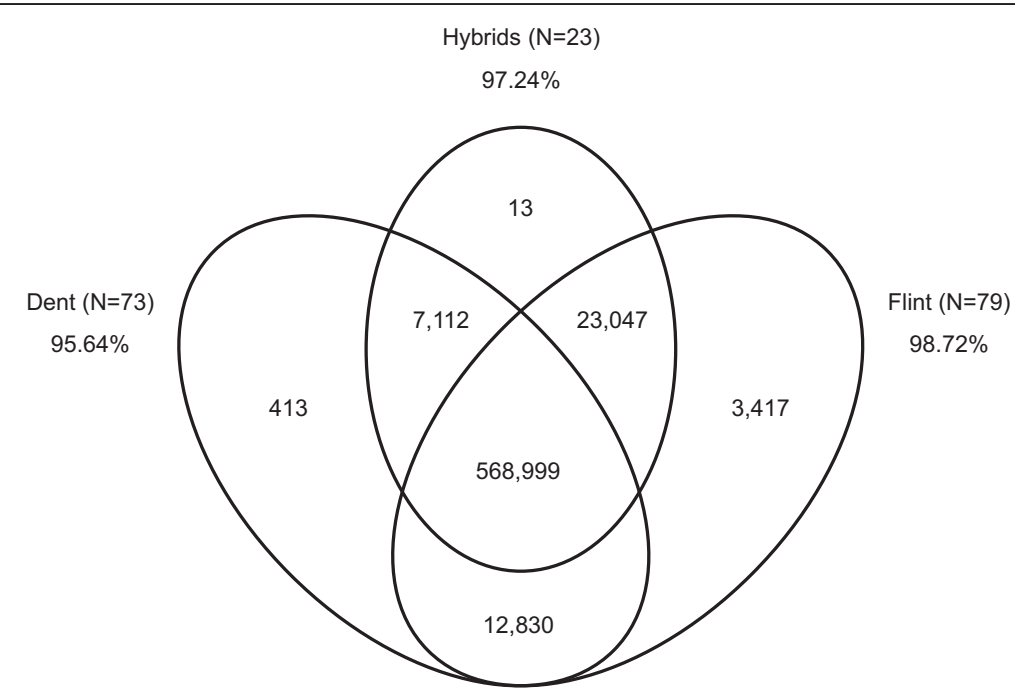

Figure 3 Polymorphic variants of the $600 \mathrm{k}$ array. Venn diagram showing the number of polymorphic variants represented on the $600 \mathrm{k}$ array in 73 Dent and 79 Flint samples and 23 hybrids of the validation panel. 

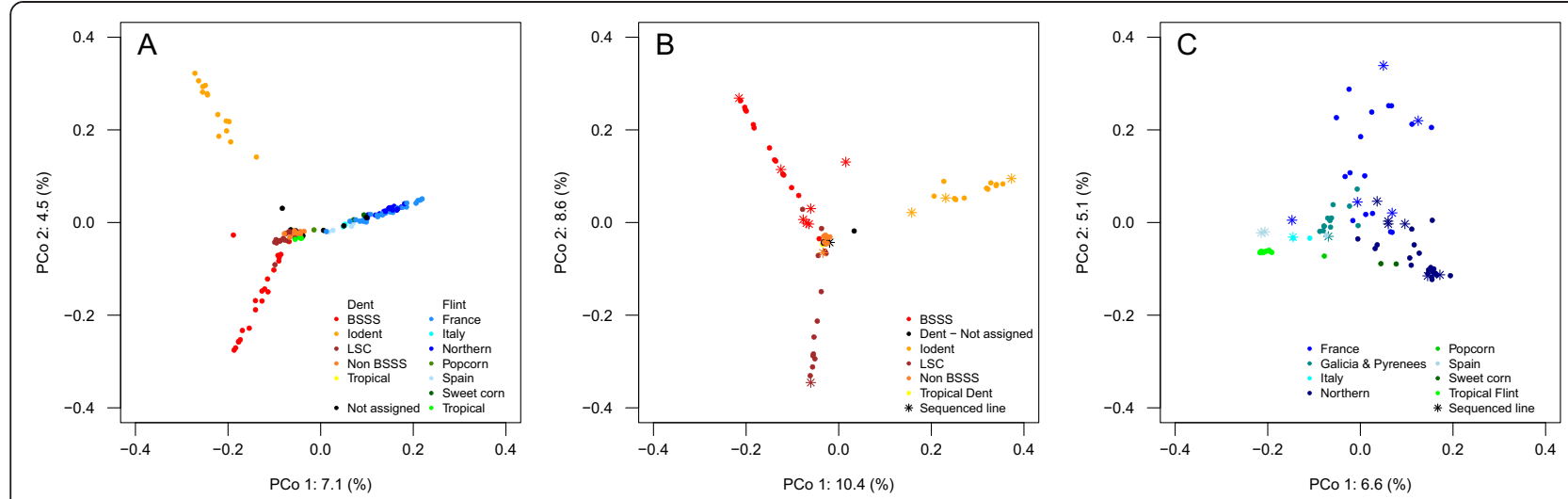

Figure 4 Population substructure in a diverse maize panel. PCoA plots of the first two axes in a diverse panel of public maize lines based on Rogers' distances from 251,152 variants including OTVs represented on the $600 \mathrm{k}$ array (markers in LD with $\mathrm{r}^{2}>0.8$ were excluded). A) Whole set of 155 maize lines, B) 73 Dent lines, and C) 79 Flint lines.

within temperate Dent, whose strong differentiation is well-known [46,47]. Compared to US material, European samples clustered within each group more towards the center (Figure 4B), suggesting a lower level of differentiation and population substructure [31].

In Central Europe, Flint plays an important role for hybrid breeding programs relying on the Dent $x$ Flint heterotic pattern. PCoA of 76 Flint lines as well as one popcorn and two sweetcorn accessions resulted in the separation of European Flint lines adapted to more Northern or Mediterranean climate, respectively (Figure 4C). This split has also been observed in other studies based on phenotypic and RFLP marker data [48] and can be traced back to the introgression of maize to Europe. Maize was introduced to Europe starting at the end of the $15^{\text {th }}$ century, when Columbus brought subtropical maize from the Caribbean Islands to Southern Spain, later followed by travelers importing so called Northern Flint [49] from Canada to Northern France [48,50-52]. The "non-Northern" Flint group in our study was further subdivided in the PCoA by the second axis depicting the relatedness of a subset of samples which had the French line F7 in their ancestry. Thus, the first two axes revealed two main subgroups of European Flint although the substructure was not as pronounced as in Dent. As indicated in Figure 4 (B, C), the sequenced lines of the variant discovery panel were nicely distributed across the different germplasm pools.

Cross-validation results obtained by ADMIXTURE [53] suggested $K=7$ as the most likely number of groups (Additional file 2: Figure S4, Additional file 2: Figure S5) with four clear clusters in Dent for BSSS, Iodents, LSC, and a mixed group of non-BSSS lines, as well as two clusters for Northern Flints and non-Northern Flints, and a mixed group of (sub)tropical lines or lines with ancestors of (sub)tropical origin. This grouping well reflects the main subgroups observed with PCoA. In accordance with an increasing sequence divergence to the reference sequence of B73, ADMIXTURE analysis based on the $46 \mathrm{k}$ flanking OTVs resulted in the subdivision of Dent, Flint, and a group including tropical lines as well as Flint lines originating from Argentina, Spain, and Italy (Additional file 2: Figure S6, Additional file 2: Figure S7).

We conclude that the variants represented on the $600 \mathrm{k}$ array are well suited for dissecting the diversity and genetic composition of temperate maize lines. Performance of the array with regard to the analysis of tropical material or wild maize relatives will need further investigation.

\section{Extent of linkage disequilibrium}

The extent of LD in a population is influenced by recombination rate, drift, mutation, selection, and population structure. It has thus influence on experimental design, resolution, and analysis of genome-wide studies. In the public inbred lines genotyped for validation, LD decay $\left(r^{2} \leq 0.2\right)$ could be observed within $158 \mathrm{~kb}$ on average with some chromosomal differences (Table 1). Group specific analysis of the LD extent revealed a substantially higher level of LD in the two Dent groups of Iodents and BSSS with mean LD decay distances of 19.5 and $36.2 \mathrm{Mb}$, respectively, compared to non-BSSS lines (excluding the LSC group) where LD decayed within $239 \mathrm{~kb}$. Due to the rather small sample size in $\operatorname{LSC}(\mathrm{N}=9)$, decay distances were not calculated for this subset. Mean LD decay values in Flint were highest for non-Northern Flints, which included several lines sharing a common ancestor, with $4.6 \mathrm{Mb}$, followed by Northern Flints (312 kb). The fastest LD decay was observed in (sub)tropical lines $(70 \mathrm{~kb})$. This corroborates previous reports supporting the close relationship and small number of founder lines within Iodent and BSSS compared to the other groups $[19,47]$. The low values of the non-BSSS as well as the 
Table 1 Mean linkage disequilibrium (LD) given as $\mathrm{r}^{2}$ and average LD decay distance ${ }^{\mathrm{a}}$ in $\mathrm{kb}$ per chromosome in 155 lines (all) and in six ${ }^{b}$ subgroups as determined by ADMIXTURE

\begin{tabular}{|c|c|c|c|c|c|c|c|c|c|c|c|c|c|c|}
\hline \multirow[b]{2}{*}{ Chr. } & \multicolumn{2}{|c|}{$\begin{array}{c}\text { All } \\
(\mathrm{N}=155)\end{array}$} & \multicolumn{2}{|c|}{$\begin{array}{c}\text { Dent - BSSS } \\
(N=14)\end{array}$} & \multicolumn{2}{|c|}{$\begin{array}{c}\text { Dent - } \\
\text { Non-BSSS } \\
(\mathrm{N}=32) \\
\end{array}$} & \multicolumn{2}{|c|}{$\begin{array}{l}\text { lodent } \\
(\mathrm{N}=14)\end{array}$} & \multicolumn{2}{|c|}{$\begin{array}{c}\text { Non-Northern } \\
\text { Flint } \\
(\mathrm{N}=18) \\
\end{array}$} & \multicolumn{2}{|c|}{$\begin{array}{l}\text { Northern Flint } \\
\qquad(\mathrm{N}=34)\end{array}$} & \multicolumn{2}{|c|}{$\begin{array}{c}\text { (Sub) Tropical } \\
(\mathrm{N}=34)\end{array}$} \\
\hline & mean $r^{2}$ & $r^{2} d r$ & nean $r^{2}$ & $r^{2}$ decay & mean $r^{2}$ & $r^{2}$ decay & mean $r^{2}$ & $r^{2}$ decay & mean $r^{2}$ & $r^{2}$ decay & mean $r^{2}$ & $\mathrm{r}^{2}$ decay & mean $r^{2}$ & $r^{2}$ decay \\
\hline 1 & 0.029 & 119.14 & 0.202 & $14,411.28$ & 0.049 & 156.32 & 0.212 & $15,949.23$ & & $4,007.83$ & 0.049 & 197.98 & 0.037 & 4338 \\
\hline 2 & 0.027 & 120.13 & 0.260 & $30,329.28$ & 0.040 & 178.63 & 0.177 & $9,411.84$ & 0.133 & $4,211.89$ & 0.039 & 332.88 & 0.039 & 04.8 \\
\hline 3 & 0.034 & 199.62 & 0.263 & $31,772.64$ & 0.057 & 306.29 & 0.170 & $8,340.20$ & 0.199 & $15,545.48$ & 0.055 & 352.29 & 0.044 & 96.2 \\
\hline 4 & 0.031 & 192.41 & 0.207 & $16,123.82$ & 0.054 & 319.43 & 0.310 & $50,389.31$ & 0.128 & $3,114.28$ & 0.063 & 404.96 & 0.037 & 838 \\
\hline 5 & 0.027 & 119.91 & 0.224 & $18,692.30$ & 0.047 & 172.69 & 0.154 & $5,681.66$ & 0.144 & $4,626.09$ & 0.056 & 262.24 & 0.038 & 57.2 \\
\hline 6 & 0.025 & 106.65 & 0.198 & $14,001.18$ & 0.049 & 188.22 & 0.170 & $8,886.16$ & 0.099 & 955.38 & 0.049 & 206.58 & 0.036 & 40.2 \\
\hline 7 & 0.033 & 176.04 & 0.214 & $16,901.24$ & 0.057 & 246.89 & 0.228 & $18,945.94$ & 0.141 & $3,737.87$ & 0.060 & 379.57 & 0.042 & 75.1 \\
\hline 8 & 0.033 & 183.72 & 0.257 & $28,937.29$ & 0.053 & 254.27 & 0.280 & $37,984.38$ & 0.120 & $1,537.13$ & 0.052 & 322.23 & 0.039 & 81.5 \\
\hline 9 & 0.033 & 167.58 & 0.309 & $53,758.98$ & 0.057 & 263.68 & 0.263 & $28,009.93$ & 0.151 & $5,020.05$ & 0.056 & 314.73 & 0.041 & 61.48 \\
\hline 10 & 0.033 & 192.64 & 0.396 & $136,831.20$ & 0.057 & 303.38 & 0.256 & $11,899.95$ & 0.130 & $2,916.32$ & 0.054 & 343.07 & 0.041 & 96.65 \\
\hline Mean & 0.031 & 158.38 & 0.253 & $36,175.92$ & 0.053 & 238.98 & 0.222 & $19,549.86$ & 0.138 & $4,567.23$ & 0.055 & 311.65 & 0.039 & 69.99 \\
\hline edian & 0.032 & 171.81 & 0.241 & $23,814.80$ & 0.054 & 250.58 & 0.220 & $13,924.59$ & 0.133 & $3,872.85$ & 0.055 & 327.55 & 0.039 & 69.70 \\
\hline
\end{tabular}

${ }^{\mathrm{a} D i s t a n c e s}$ in $\mathrm{kb}$ for $\mathrm{r}^{2}=0.2$ calculated per $50 \mathrm{Mb}$ window.

${ }^{b} \mathrm{LD}$ decay distances were not calculated for $\mathrm{LSC}(\mathrm{N}=9)$.

(sub)tropical lines in our study might be explained by the high heterogeneity of both groups. Still, LD levels in our panel of maize lines were higher compared to previous studies reporting the breakdown of LD within distances between 5 and $10 \mathrm{~kb}[18,54]$ in highly diverse maize lines. The higher LD extent in our study might be due to the sample panel analysed, which mainly comprised temperate elite maize inbred lines belonging to distinct germplasm pools but no landraces or wild species. The variants selected for the $600 \mathrm{k}$ array fulfill the requirements by [55], after which genotyping arrays should have sufficient coverage to capture the fastest LD decay of the considered heterotic pools. Thus, especially for analysis of diverse sample panels, high density genotyping arrays are of interest for estimating global and local LD.

\section{Other potential applications of the maize high density array}

We presented the usefulness of the maize high density $600 \mathrm{k}$ array for the analysis of population structure and LD, but of course it is suitable for many other applications in maize research and breeding. For population genetic analyses based on genotyping data ascertainment bias is a central aspect [56]. The lines sequenced in this study were chosen to represent the diversity within the more comprehensive validation panel. However, bias may be introduced by the filtering steps that are applied during array development and typically results in a bias towards intermediate allele frequencies. Flanking OTVs have the advantage of not being directly targeted by the variant filtering procedure itself offering thus the potential to counteract ascertainment bias [40]. Compared to the minor allele frequency (MAF) distribution of the complete set of variants of the $600 \mathrm{k}$ array where only $3.8 \%$ of the variants detect rare alleles with a MAF $<0.05$, OTVs showed with $40.8 \%$ rare alleles $(\mathrm{MAF}<0.05)$ a reduced bias towards intermediate allele frequencies (Figure 5). Thus, even if the specific type of the variant as well as its exact location is unknown, flanking OTVs

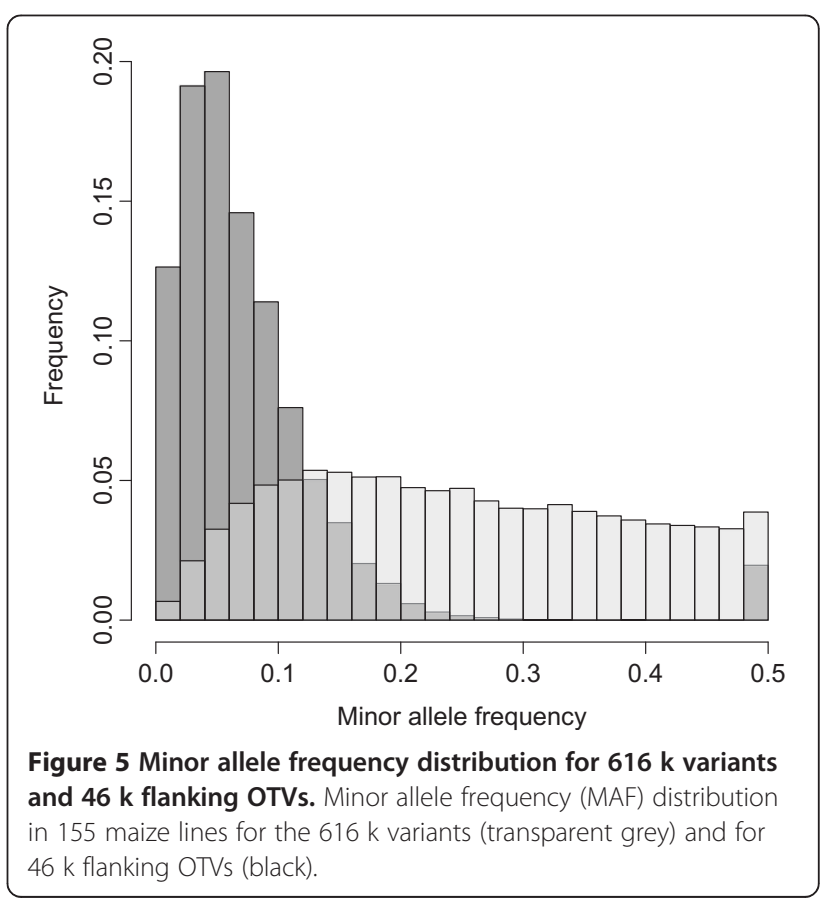


represent an interesting subset of variants for population genetic analyses like screens for selection signatures based on genotyping data which we will address in further studies.

Further applications of the $600 \mathrm{k}$ array include its use in genome-wide and targeted approaches. The array should have the desired density for genome-wide association studies in maize, for which the currently available density of the Illumina MaizeSNP50 BeadChip was shown to provide limited resolution [57]. Due to the extremely high marker density, the array can be used for bulked segregant analysis to identify genomic regions involved in phenotypic traits with monogenic or oligogenic inheritance [58]. Further applications may be seen in the context of plant variety protection and in the investigation of pedigree relationships, identity-by-descent regions and ancestral lineages [59]. A panel of representative lines genotyped with the $600 \mathrm{k}$ array should also allow high accuracy in imputation of genotypes from genetic material analysed with lower density marker panels [60]. Finally, custom sets of SNPs may be assembled from any genomic region and converted into other highly flexible SNP assay formats to saturate specific regions in fine-mapping, mapbased cloning studies or marker-assisted selection, since flanking sequence information is available and conversion rates among SNP platforms are generally high.

\section{Conclusions}

This paper describes the establishment of the currently largest publicly available SNP array in crop species composed of 616,201 SNPs and small indels. The Affymetrix ${ }^{\circ}$ Axiom ${ }^{\bullet}$ Maize Array is optimized for European and American temperate maize. It is well suited for finemapping of genomic regions, genome-wide studies, and detection of marker-trait associations. Important aspects in the development of the maize $600 \mathrm{k}$ genotyping array were: (i) identification of polymorphic, high-confidence variants based on whole genome sequence data of 30 representative temperate maize lines, (ii) selection of physically equally distributed variants for validation, taking predicted variant performance and subgroup specific segregation into account, (iii) experimental variant validation by genotyping 285 DNA samples originating from diverse subgroups of maize, and (iv) final selection of variants upon stringent filtering based on cluster metrics, concordance with in silico calls, and physical position. We have shown that the variants selected for the $600 \mathrm{k}$ genotyping array were polymorphic in a broad maize panel, ensuring its suitability for a wide range of applications. We investigated a subset of variants (OTVs) that showed almost no bias towards intermediate allele frequencies, thus are potentially of interest for population genetic analyses. Finally, we performed principal coordinate, admixture, and LD analyses to illustrate the potential of the array to analyse population substructure and LD decay with high resolution.

\section{Methods}

\section{Sequencing of $\mathbf{3 0}$ maize lines in the variant discovery} panel

For whole genome sequencing of 30 maize inbred lines (Additional file 1: Table S1), DNA was extracted from leaf material frozen in liquid nitrogen following the protocol of [61]. For deep-sequencing of the three Flint lines DK105, EP1, and F7, as well as the Dent line PH207, DNA was extracted from a single plant each. For sequencing of the other 26 maize lines, DNA was extracted from bulked leaf samples of 8-10 plants per line. Sequencing was performed on an Illumina ${ }^{\bullet}$ HiSeq 2000 platform, generating $2 \times 100 \mathrm{bp}$ paired-end reads from standard 300 bp libraries using manufacturer's protocols.

\section{In silico variant discovery and pre-selection}

Sequence reads were mapped against reference sequence B73 AGP_v2 using BWA (version 0.7.5) [62]. Alignments were post-processed by marking duplicates and fixing paired-end information applying the PICARD toolbox (version 1.84) [63] and by performing local realignments using the Genome Analysis Toolkit (GATK, version 2.4-9) [35]. Quality scores of called variant positions (SNPs and short insertions/deletions) were improved by recalibrating values according to results of several covariate analyses (homopolymer, cycle, dinucleotide, quality score) done on a set of trusted variants. As no SNP database was available for the maize varieties under study, a database of high quality trusted SNPs was created following the recommendation on the GATK website (http://www.broadinstitute.org/gatk/guide/tagged?tag=baserecalibrator).

Briefly, initial variants were called independently using two algorithms to obtain a more robust SNP set, as recommended by [64]. We used SAMtools (version 0.1.18) [34] and the intersection to the initial GATK variants was further filtered for SNP quality ( $\geq 50$ ); low and excessive read coverage $(50 \leq \mathrm{DP}<3000)$; presence of the reference allele; and homozygous non-reference calls in at least two of the 30 lines. In a second round, variants were identified from the base quality recalibrated bam files by the GATK Unified Genotyper. For the final set of candidate variants, several stringent filters were applied. First, variants were excluded if they were located in regions with genomic copy number $\geq 50$ (based on 16 -kmer counts). Second, variants were not forwarded to the next step if (i) more than $5 \%$ of reads had mapping quality 0, (ii) coverage was more than six fold higher compared to the mean coverage, or (iii) a SNP quality score was below 100. In addition, variants had to exhibit a minimal distance of 20 bp between neighboring variants located in at least one flanking sequence. In 
summary, the final pre-selection variant set scored for tiling by the Affymetrix ${ }^{\circledast}$ Axiom $^{\oplus}$ myDesign GW bioinformatics pipeline comprised a total of 5,641,493 bi-allelic variants. For annotation of the variants, version $5 \mathrm{~b} 60$ of the reference sequence B73 AGP_v2 was used (ftp://ftp. gramene.org/pub/gramene/maizesequence.org/release-5b/ filtered-set/) which contains 39,656 gene models. Variant effects were predicted using SNPeff (version 3.2) [65].

\section{Variant selection for the screening arrays according to predicted conversion quality, physical position, and segregation in Dent and Flint}

For all variants from the $5.6 \mathrm{M}$ list $\mathrm{p}$-convert values were calculated per probe according to the Affymetrix ${ }^{\oplus}$ Axiom myDesign GW bioinformatics pipeline and categorized as "recommended", "neutral", "not recommended", and "not possible", respectively. The p-convert value can take a value between 0 and 1 and describes the predicted probability to convert on the array by taking its sequence, binding energies, expected degree of nonspecific binding and hybridization to multiple genomic regions into account. Two probe sets (forward and reverse) for each SNP from the Illumina MaizeSNP50 BeadChip (GenTrain score $>0$ ) were directly included in the list of variants for the screening arrays without further filtering unless they were classified as "not possible". For the newly identified variants only probe sets categorized as "recommended" or "neutral" were further analyzed. For coding variants, the probe with the higher p-convert value was chosen based on this classification, whereas for all remaining variants probe sets were further filtered according to the following multi step approach. Based on the reference genome size of 2.066 $\mathrm{Gb}$, first, the maize genome was partitioned in 20,660 bins of size $100 \mathrm{~kb}$, aiming at an equal physical distribution of variants. Assuming up to $1.23 \mathrm{M}$ possible variants, which could be tested on two screening arrays, after substracting the fixed variants $(150 \mathrm{k}$ coding variants and $2 * 48 \mathrm{k}$ Illumina ${ }^{\circ}$ MaizeSNP50 BeadChip SNPs), each $100 \mathrm{~kb}$ bin would contain on average 48 variants. Three cases were distinguished to fill the physical bins: (i) all possible variants of a bin were included if less than 48 "recommended" or "neutral" variants were identified in the corresponding bin, (ii) "recommended" variants were considered as fixed, if their number did not exceed 48 and remaining "neutral" variants were subjected to another filtering step, and (iii) "recommended" variants were further filtered, if $\geq 48$ were observed in the corresponding bin. For this filtering step to fill up underrepresented bins, allele frequencies were determined for Dent $(\mathrm{N}=13)$ and Flint $(\mathrm{N}=17)$ lines separately by calculating the ratio of homozygous non-reference allele calls in relation to all available calls per variant. Variants were classified according to their pool-specific allele frequency as class "A", corresponding to intermediate (between 0.2 and 0.8 ), or " $\mathrm{B}$ " to extreme non-reference allele frequencies $(<0.2$ or $>0.8)$. One third of the variants, which filled up the bins, were chosen to be specific for Flint (category Dent "A" | Flint "B") and one third specific for Dent (Dent "B" | Flint "A"), respectively. Further, one sixth each had to be either common (category Dent "A" | Flint "A") or rare for both groups (Dent "B" | Flint "B"), respectively.

In a final step, $50 \mathrm{~kb}$ bins were considered if the original $100 \mathrm{~kb}$ bin had been filled with less than 48 variants. Additional variants were selected if there were less than 8 variants per $50 \mathrm{~kb}$ bin to avoid underrepresentation of genomic regions by choosing variants randomly (i) from "recommended" variants with at least six, but less than 22 homozygous reference allele calls in at least 28 of the 30 lines of the discovery panel to avoid extreme allele frequencies (maximal six variants per $50 \mathrm{~kb}$ bin), and (ii) if further variants were required, from all remaining variants. Altogether, a final list of 1,228,506 variants was established for validation with a diverse panel of maize lines on two customized $675 \mathrm{k}$ Affymetrix ${ }^{\circ}$ Axiom $^{\oplus}$ myDesign GW screening arrays.

\section{Plant material for genotyping}

The selected set of $1.2 \mathrm{M}$ variants was used to genotype 285 DNA samples from genetically diverse maize germplasm to evaluate their assay performance. The validation panel was composed of 224 Dent and Flint inbred lines of which 92 were proprietary lines. From those, line B37 was included three times as technical replicate and three lines (B73, DK105, EP1) were represented by two biological replicates each. In addition, 13 tropical lines (ten Flint, three Dent), ten doubled haploid lines from three European Flint landraces, four lines with no available pool assignment, and two Teosinte accessions were analysed. Finally, we included 27 hybrids, among which there were 23 F1 hybrids from Mendelian trios with both parental lines present in the public elite line panel, and four proprietary hybrids (Additional file 1: Table S4). The Dent elite lines comprised representative samples belonging to the subgroups Iowa Stiff Stalk Synthetic (BSSS), Lancaster Sure Crop (LSC), or Iodent, as well as other non-BSSS samples, and samples with tropical origin. The Flint panel was composed of European Northern Flints and lines originating from Spain, Italy, and France, as well as sweetcorn, popcorn, and tropical lines. Except for the 92 proprietary inbred lines, the elite inbred lines were selected according to their frequency of use and citation $[46,47]$ as well as based on utilization in other studies, pedigree information or classifcation available from literature $[66,67]$ or from internet sources [68] with the aim to represent diverse temperate material. The 96 proprietary samples were included in the analysis of the screening 
array for training of the variant clustering algorithm, but not in further analyses presented here.

DNA for genotyping was extracted from seeds available to the authors or kindly provided by the following institutions: INRA UMR de Génétique Végétale (Gif-sur-Yvette, France), Universität Hohenheim (Stuttgart, Germany), USDA-ARS (Ames, USA), CIAM (La Coruña, Spain), CRA-MAC Maize Research Unit (Bergamo, Italy), and CSIC (Pontevedra, Spain).

\section{Comparison of variant calls with the Illumina ${ }^{\circledR}$ MaizeSNP50 BeadChip}

The 30 sequenced lines (Additional file 1: Table S1) were genotyped with the Illumina ${ }^{\circ}$ MaizeSNP50 BeadChip following manufacturer's protocols using a total of $50 \mathrm{ng}$ genomic DNA. Raw hybridization intensity data processing, clustering, and genotype calling were performed using the software GenomeStudio (v2011.1, Illumina ${ }^{\circ}$ ) and the public cluster file II described in [21].

\section{Experimental variant validation by genotyping}

From each sample, $200 \mathrm{ng}$ genomic DNA per array was used for analysis on the Affymetrix GeneTitan ${ }^{\bullet}$ platform with the Axiom myDesign GW genotyping array following manufacturer's protocol. After array processing, four samples were excluded from further analyses as signal intensity files could be created for only one of the two screening arrays, resulting in 281 samples remaining for further investigation (Additional file 1: Table S4).

Raw hybridization intensity data processing, clustering, genotype calling (genotypes $\mathrm{AA}, \mathrm{AB}, \mathrm{BB}$ ), off-target variant (OTV; genotypes $\mathrm{AA}, \mathrm{AB}, \mathrm{BB}, \mathrm{OO}$ ) calling, and variant categorization according to genotype cluster metrics (Additional file 2: Figure S2) were performed using Affymetrix Power Tools (APT, version 1.15.0) and the package SNPolisher (version 1.3.6.6) [69] for R (version 3.0.1) [70] according to the Axiom Genotyping Solution Data Analysis Guide. For initial genotype calling generic $a$ priori cluster positions were used since no information about expected cluster positions was available. The three possible genotype clusters were then redefined in a posteriori cluster positions, taking the observed genotype call positions into account and variants were finally classified according to selected cluster metrics. A first analysis was performed according to the recommendations of Affymetrix, but with a reduced threshold (0.90) for the variant call frequency instead of the default value (0.97) to account for the high amount of PAVs in the maize genome [17].

In a second, extended analysis different levels of inbreeding were taken into account for a posteriori cluster definition because of the high amount of lines in the validation panel exhibiting only a small proportion of heterozygosity in contrast to populations in Hardy-Weinberg equilibrium. The inbred correction was achieved by a parameter assigning sample-specific penalties using the "-read-inbred" parameter for the "apt-probeset-genotype" command in APT. This parameter takes values from 0 for fully heterozygous to 16 for completely homozygous samples and includes this information for re-defining a priori cluster positions for genotype calling. We assigned values of 0 for F1 hybrids, 12 for inbreds with unclear homozygosity level, and 14 for pure inbred and doubled haploid lines to allow some remaining heterozygosity (Additional file 1: Table S4). Results of the analyses with and without inbred correction were compared and a subset of randomly selected genotype clusters were visually checked.

\section{Selection of high-confidence variants for construction of the final $600 \mathrm{k}$ array}

Variants were preferentially selected if they were exhibiting stable category assignments (Additional file 2: Figure S2) with clearly separated clusters to avoid restrictions dependent on the inbred-level. Categories were assigned by the classification step of SNPolisher using the following parameters: CR.cut $=90, \mathrm{FLD}$.cut $=3.6$, HetSO.cut $=-0.1$, HetSO.OTV.cut $=-0.3$, HomRO2. cut $=0.3, \quad$ HomRO3.cut $=-0.9, \quad$ HomRO.flag $=$ TRUE, nMinorAllele.cut $=2$. For high quality variant selection, a total of 523,154 variants classified as "PolyHighResolution" (PHR) with and without inbred correction were directly forwarded to the final list as they were characterized by distinct and narrow clusters in both analyses. These variants were used to define customized cluster quality criteria for OTVs to ensure a clear separation of genotype clusters, but allowing in addition lower heterozygous cluster signal intensities due to cluster splits caused by unexpected off-target variants in the flanking region of the target variant or potential tri-allelic variants. The "Fisher's Linear Discriminant" (FLD) value characterized the cluster quality being highest in case of well-separated and narrow clusters. The "Heterozygous Cluster Strength Offset" (HetSO) measured the difference in the signal intensities of the genotype clusters as the heterozygous cluster should have higher signal intensity on average compared to the homozygous ones due to technical features of the array. The "Homozygote Ratio Offset" (HomRO) described the distance of the homozygous clusters to the heterozygous one to detect potentially misplaced clusters. The chosen thresholds upon inbred correction were the following: no monomorphic variants, $\leq 10 \%$ missing calls (corresponding to $\leq 30$ missing calls), FLD $>3.5$, HetSO $>-3.5$, and HomRO $>1$. As FLD and HetSO values were exhibiting missing values in some variants with only two clusters, an additional threshold was set in this case using a FLD value between homozygous clusters (homFLD) of $>5$. All 42,877 variants which were classified in both analyses (with and without inbred correction) as OTV and passed in addition 
the above thresholds were included in the selection for the final array.

Remaining variants were ranked by applying a voting system. First, variants were ranked according to their classification with and without inbred correction. Variants, which were classified as "OTVstable" or changed their category from "NoMinorHom" (only one homozygous and one heterozygous genotype cluster) without inbred correction to PHR after inbred correction, were assigned a weight of 10 . Variants, which belonged to any other class without inbred correction, but changed to PHR after inbred correction received a weight of 5 , and all remaining variants not fulfilling the previous criteria obtained a weight of 0 . Second, variants were weighted regarding the concordance of their calls with the in silico variant calls from sequencing. The number of matching calls per variant across the 30 sequenced lines from the discovery panel, which all were also analyzed on the genotyping test arrays, was normalized to the total number of calls per variant resulting in a value in the range of 0 to 1 . As a third criterion, the over- or underrepresentation of the corresponding $100 \mathrm{~kb}$ bin was taken into account by calculating the deviation of the number of variants in the corresponding bin to the mean of variants in the five bins up- and downstream, respectively, and scaling the value into a range of values between -1 and 1. For the final rank, the sum was built of (i) the weight of the variant class that was multiplied with 35 to ensure a high performance on the final array (range: 0 to 750), (ii) the value of the sequence match multiplied with 90 to minimize false-positives (range: 0 to 90), and (iii) the weight of the bin representation, which was multiplied by 10 for lowest impact (range: -10 to 10 ). For the 48,324 Illumina ${ }^{\circledR}$ MaizeSNP50 SNPs which were tiled from both sides, the probe with the higher rank was included in the final set in case of varying ranks. If both probes of a variant exhibited the same rank, one probe was chosen randomly. Due to an erroneous mapping of 2,669 Illumina MaizeSNP50 BeadChip SNPs to the B73 reference sequence a wrong (non-polymorphic) position was assayed on the screening arrays and these non-validated SNPs were not included on the final array. The top 616,201 variants were selected for the final array design among which 45,655 originated from the Illumina MaizeSNP50 BeadChip. Information on SNP IDs, genome positions, probe sets, and alleles are available at NCBI GEO as platform GPL18778 (http://www.ncbi.nlm.nih. gov/geo/query/acc.cgi?acc=GPL18778) or from the product information of manufacturer Affymetrix.

\section{Analyses of population substructure and linkage disequilibrium}

For all analyses indels were treated as bi-allelic SNPs. In PCoA and ADMIXTURE analyses OTVs were included with their genotype calls of the target variant as well as information on presence or absence of a flanking variant, resulting in $616 \mathrm{k}$ plus $46 \mathrm{k}$ variants. Variants with $\geq 10 \%$ of missing data were excluded. Remaining missing data were imputed using Beagle [71] via the R package "synbreed" [72] with $\mathrm{R}$ version 3.0.1 [70]. Public inbreds $(\mathrm{N}=155$, replicates excluded) of the validation panel were investigated with $\mathrm{PCoA}$ and ADMIXTURE for population structure as well as for LD decay. LD pruning was performed for PCoA and ADMIXTURE analyses by applying a $\mathrm{r}^{2}$ threshold of 0.8 . PCoA based on Rogers' distances was performed using $\mathrm{R}$ with the packages "synbreed" [72], "adegenet" [73], and "ape" [74]. Analysis of population substructure was calculated using ADMIXTURE (version 1.23) [53] running with default settings for $K=1$ to $K=15$. LD was calculated chromosome-wise per $50 \mathrm{Mb}$ window using Plink (version 1.07) [75] and LD decay analysis was performed using the R package "synbreed" [72].

\section{Availability of supporting data}

Supporting sequence data are available in the NCBI Sequence Read Archive (SRA) repository under BioProject accession number PRJNA260788 (http://www.ncbi.nlm. nih.gov/bioproject/PRJNA260788). Information on SNP IDs, genome positions, probe sets, and alleles can be retrieved from NCBI GEO, platform GPL18778 (http:// www.ncbi.nlm.nih.gov/geo/query/acc.cgi?acc=GPL18778).

\section{Additional files}

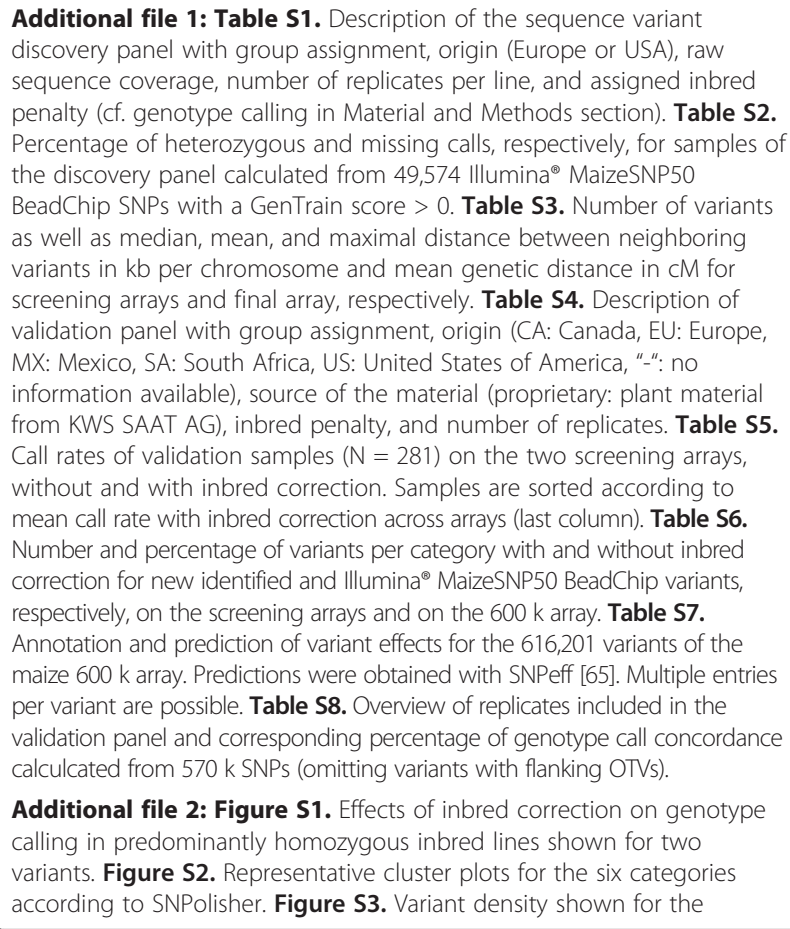

Additional file 2: Figure S1. Effects of inbred correction on genotype calling in predominantly homozygous inbred lines shown for two variants. Figure S2. Representative cluster plots for the six categories according to SNPolisher. Figure S3. Variant density shown for the 
screening arrays (light grey) and for the variants of the Affymetrix ${ }^{\oplus}$ Axiom ${ }^{\oplus}$ Maize Array (black) across the 10 maize chromosomes. Centromere positions are indicated by a black horizontal bar. Figure S4. Cross-validation errors from ADMIXTURE for different values of $K$ for 155 maize lines based on 251,152 variants including OTVs (markers in LD with $r^{2}>0.8$ were excluded). Figure S5. Subgroups identified in 155 maize lines of the validation panel as revealed by ADMIXTURE for $\mathrm{K}=7$ based on 251,152 variants including OTVs (markers in LD with $r^{2}>0.8$ were excluded). Figure S6. Cross-validation errors from ADMIXTURE for different values of $\mathrm{K}$ for 155 maize lines based on 27,099 flanking OTVs (markers in LD with $r^{2}>0.8$ were excluded). Figure S7. Subgroups identified in the 155 public lines of the validation panel as revealed by admixture for $\mathrm{K}=3$ based on 27,099 flanking OTVs (markers in LD with $r^{2}>0.8$ were excluded)

\section{Competing interests}

Two commercial organisations - KWS SAAT AG and Affymetrix Inc. - were involved in the development of the array and in preparation of the manuscript. Among the authors, CK and MO work for KWS SAAT AG, and AD and CB work for Affymetrix.

\section{Authors' contributions}

This study was carried out in collaboration between all authors. CCS, EB, GH, KFXM, and MO conceived the study; CK and EB provided DNA for sequencing and genotyping as well as information on the maize lines; TM and TMS sequenced the DNA samples of the discovery panel; GH and MS carried out variant calling and bioinformatic analyses; CB performed the Affymetrix ${ }^{\oplus}$ Axiom ${ }^{\oplus}$ design; CCS, CK, EB, GH, MS, and SU developed and discussed the variant selection strategy and discussed results; HP and RF processed the genotyping arrays; AD helped with the genotype calling pipeline; $E B, G H, M S$, and SU analyzed the genotypic data; EB and SU drafted the manuscript; $\mathrm{CCS}, \mathrm{GH}$, and MS edited the manuscript and all authors read and approved the final manuscript.

\section{Acknowledgements}

We are grateful to Alain Charcosset (INRA UMR de Génétique Végétale, Gif-sur-Yvette, France), Albrecht E. Melchinger (Universität Hohenheim, Institute of Plant Breeding, Seed Science and Population Genetics, Stuttgart, Germany), Mark J. Millard (USDA-ARS, Ames, USA), Jesús Moreno González (CIAM, La Coruña, Spain), Rita Redaelli (CRA-MAC Maize Research Unit, Bergamo, Italy), Pedro Revilla (CSIC, Pontevedra, Spain), and Karl Schmid (Universität Hohenheim, Crop Plant Biodiversity and Breeding Informatics) for providing seeds of maize lines and information about pedigrees and group assignment. This study was funded by the Federal Ministry of Education and Research (BMBF, Germany) within the AgroClustEr Synbreed - Synergistic plant and animal breeding (grant 0315528) and publication was supported by the German Research Foundation (DFG) and the Technische Universität München within the funding programme Open Access Publishing.

\section{Author details}

'Plant Breeding, Centre of Life and Food Sciences Weihenstephan, Technische Universität München, 85354 Freising, Germany. ${ }^{2}$ Plant Genome and System Biology, Helmholtz Zentrum München, 85764 Neuherberg, Germany. ${ }^{3}$ KWS SAAT AG, 37555 Einbeck, Germany. ${ }^{4}$ Institute of Human Genetics, Helmholtz Zentrum München, 85764 Neuherberg, Germany. ${ }^{5}$ Animal Breeding, Centre of Life and Food Sciences Weihenstephan, Technische Universität München, 85354 Freising, Germany. ${ }^{6}$ Affymetrix Inc., Santa Clara, CA 95051, USA.

Received: 11 June 2014 Accepted: 23 September 2014 Published: 29 September 2014

\section{References}

1. Ragoussis J: Genotyping technologies for genetic research. Annu Rev Genomics Hum Genet 2009, 10:117-133.

2. Hayes BJ, Lewin HA, Goddard ME: The future of livestock breeding: genomic selection for efficiency, reduced emissions intensity, and adaptation. Trends Genet 2013, 29(4):206-214.

3. Langridge P, Fleury D: Making the most of 'omics' for crop breeding. Trends Biotechnol 2011, 29(1):33-40.
4. Zhao K, Tung CW, Eizenga GC, Wright MH, Ali ML, Price AH, Norton GJ, Islam MR, Reynolds A, Mezey J, McClung AM, Bustamante CD, McCouch SR: Genome-wide association mapping reveals a rich genetic architecture of complex traits in Oryza sativa. Nat Commun 2011, 2:467.

5. Myocardial Infarction Genetics Consortium: Genome-wide association of early-onset myocardial infarction with single nucleotide polymorphisms and copy number variants. Nat Genet 2009, 41(3):334-341.

6. Atwell S, Huang YS, Vilhjalmsson BJ, Willems G, Horton M, Li Y, Meng D, Platt A, Tarone AM, Hu TT, Jiang R, Muliyati NW, Zhang X, Amer MA, Baxter I, Brachi B, Chory J, Dean C, Debieu M, de Meaux J, Ecker JR, Faure N, Kniskern JM, Jones JDG, Michael T, Nemri A, Roux F, Salt DE, Tang C, Todesco M: Genome-wide association study of 107 phenotypes in Arabidopsis thaliana inbred lines. Nature 2010, 465(7298):627-631.

7. Hackett CA, McLean K, Bryan GJ: Linkage analysis and QTL mapping using SNP dosage data in a tetraploid potato mapping population. PLOS ONE 2013, 8(5):e63939.

8. Chen H, Xie W, He H, Yu H, Chen W, Li J, Yu R, Yao Y, Zhang W, He Y, Tang $X$, Zhou F, Deng XW, Zhang Q: A high-density SNP genotyping array for rice biology and molecular breeding. Mol Plant 2014, 7(3):541-553.

9. Meuwissen THE, Hayes BJ, Goddard ME: Prediction of total genetic value using genome-wide dense marker maps. Genetics 2001, 157(4):1819-1829.

10. Rincent R, Laloe D, Nicolas S, Altmann T, Brunel D, Revilla P, Rodriguez VM, Moreno-Gonzalez J, Melchinger A, Bauer E, Schön CC, Meyer N, Giauffret C, Bauland C, Jamin P, Laborde J, Monod H, Flament P, Charcosset A, Moreau L: Maximizing the reliability of genomic selection by optimizing the calibration set of reference individuals: comparison of methods in two diverse groups of maize inbreds (Zea mays L.). Genetics 2012, 192(2):715-728.

11. Qanbari S, Pimentel ECG, Tetens J, Thaller G, Lichtner P, Sharifi AR, Simianer H: A genome-wide scan for signatures of recent selection in Holstein cattle. Anim Genet 2010, 41(4):377-389.

12. McEvoy BP, Montgomery GW, McRae AF, Ripatti S, Perola M, Spector TD, Cherkas L, Ahmadi KR, Boomsma D, Willemsen G, Hottenga JJ, Pedersen NL, Magnusson PKE, Kyvik KO, Christensen K, Kaprio J, Heikkilä K, Palotie A, Widen E, Muilu J, Syvänen A-C, Liljedahl U, Hardiman O, Cronin S, Peltonen L, Martin NG, Visscher PM: Geographical structure and differential natural selection among North European populations. Genome Res 2009, 19(5):804-814.

13. van Heerwaarden J, Doebley J, Briggs WH, Glaubitz JC, Goodman MM, Gonzalez JDS, Ross-lbarra J: Genetic signals of origin, spread, and introgression in a large sample of maize landraces. Proc Natl Acad Sci U S A 2011, 108(3):1088-1092.

14. Ahn S, Tanksley SD: Comparative linkage maps of the rice and maize genomes. Proc Natl Acad Sci U S A 1993, 90(17):7980-7984.

15. Schnable PS, Ware D, Fulton RS, Stein JC, Wei F, Pasternak S, Liang C, Zhang J, Fulton L, Graves TA, Minx P, Reily AD, Courtney L, Kruchowski SS, Tomlinson C, Strong C, Delehaunty K, Fronick C, Courtney B, Rock SM, Belter E, Du FY, Kim K, Abbott RM, Cotton M, Levy A, Marchetto P, Ochoa K, Jackson SM, Gillam B, et al: The B73 maize genome: complexity, diversity, and dynamics. Science 2009, 326(5956):1112-1115.

16. Schnable JC, Springer NM, Freeling M: Differentiation of the maize subgenomes by genome dominance and both ancient and ongoing gene loss. Proc Natl Acad Sci U S A 2011, 108(10):4069-4074.

17. Springer NM, Ying K, Fu Y, Ji TM, Yeh CT, Jia Y, Wu W, Richmond $T$, Kitzman J, Rosenbaum $H$, Iniguez AL, Barbazuk WB, Jeddeloh JA, Nettleton D, Schnable PS: Maize inbreds exhibit high levels of copy number variation (CNV) and presence/absence variation (PAV) in genome content. PLoS Genet 2009, 5(11):e1000734.

18. Chia J-M, Song C, Bradbury PJ, Costich D, de Leon N, Doebley J, Elshire RJ, Gaut B, Geller L, Glaubitz JC, Gore M, Guill KE, Holland J, Hufford MB, Lai J, Li M, Liu X, Lu Y, McCombie R, Nelson R, Poland J, Prasanna BM, Pyhajarvi T, Rong T, Sekhon RS, Sun Q, Tenaillon MI, Tian F, Wang J, Xu X, et al: Maize HapMap2 identifies extant variation from a genome in flux. Nat Genet 2012, 44(7):803-807

19. Romay MC, Millard MJ, Glaubitz JC, Peiffer JA, Swarts KL, Casstevens TM, Elshire RJ, Acharya CB, Mitchell SE, Flint-Garcia SA, McMullen MD, Holland JB, Buckler ES, Gardner CA: Comprehensive genotyping of the USA national maize inbred seed bank. Genome Biol 2013, 14(6):R55.

20. Elshire RJ, Glaubitz JC, Sun Q, Poland JA, Kawamoto K, Buckler ES, Mitchell SE: A robust, simple genotyping-by-sequencing (GBS) approach for high diversity species. PLOS ONE 2011, 6(5):e19379.

21. Ganal MW, Durstewitz G, Polley A, Berard A, Buckler ES, Charcosset A, Clarke JD, Graner EM, Hansen M, Joets J, Le Paslier MC, McMullen MD, Montalent 
P, Rose M, Schön CC, Sun Q, Walter H, Martin OC, Falque M: A large maize (Zea mays L.) SNP genotyping array: development and germplasm genotyping, and genetic mapping to compare with the B73 reference genome. PLOS ONE 2011, 6(12):e28334.

22. Li H, Peng $Z Y$, Yang $X H$, Wang WD, Fu JJ, Wang JH, Han YJ, Chai YC, Guo $\pi$, Yang $N$, Liu J, Warburton ML, Cheng YB, Hao XM, Zhang P, Zhao JY, Liu YJ, Wang GY, Li JS, Yan JB: Genome-wide association study dissects the genetic architecture of oil biosynthesis in maize kernels. Nat Genet 2013, 45(1):43-50.

23. Gresset $\mathrm{S}$, Westermeier $\mathrm{P}$, Rademacher $\mathrm{S}$, Ouzunova M, Presterl $\mathrm{T}$, Westhoff $\mathrm{P}$, Schön C-C: Stable carbon isotope discrimination is under genetic control in the C4 species maize with several genomic regions influencing trait expression. Plant Physiol 2014, 164(1):131-143.

24. Albrecht T, Auinger HJ, Wimmer V, Ogutu JO, Knaak C, Ouzunova M, Piepho HP, Schön C-C: Genome-based prediction of maize hybrid performance across genetic groups, testers, locations, and years. Theor Appl Genet 2014, 127(6):1375-1386.

25. Hufford MB, Lubinksy P, Pyhajarvi T, Devengenzo MT, Ellstrand NC, Ross-lbarra J. The genomic signature of crop-wild introgression in maize. PLoS Genet 2013, 9(5):e1003477.

26. Song QJ, Hyten DL, Jia GF, Quigley CV, Fickus EW, Nelson RL, Cregan PB: Development and evaluation of SoySNP50K, a high-density genotyping array for soybean. PLOS ONE 2013, 8(1):e54985.

27. Wang S, Wong D, Forrest K, Allen A, Chao S, Huang BE, Maccaferri M, Salvi S, Milner SG, Cattivelli L, Mastrangelo AM, Whan A, Stephen S, Barker G, Wieseke R, Plieske J, International Wheat Genome Sequencing C, Lillemo M, Mather D, Appels R, Dolferus R, Brown-Guedira G, Korol A, Akhunova AR, Feuillet C, Salse J, Morgante M, Pozniak C, Luo MC, Dvorak J, et al: Characterization of polyploid wheat genomic diversity using a high-density 90,000 single nucleotide polymorphism array. Plant Biotechnol J 2014, 12(6):787-796.

28. Kranis A, Gheyas AA, Boschiero C, Turner F, Yu L, Smith S, Talbot R, Pirani A Brew F, Kaiser P, Hocking P, Fife M, Salmon N, Fulton J, Strom T, Haberer G, Weigend S, Preisinger R, Gholami M, Qanbari S, Simianer H, Watson K, Woolliams J, Burt D: Development of a high density 600K SNP genotyping array for chicken. BMC Genomics 2013, 14:59.

29. Rincon G, Weber KL, Eenennaam AL, Golden BL, Medrano JF: Performance of bovine high-density genotyping platforms in Holsteins and Jerseys. J Dairy Sci 2011, 94(12):6116-6121.

30. Matukumalli LK, Schroeder S, DeNise SK, Sonstegard TS, Lawley CT, Georges M, Coppieters W, Gietzen K, Medrano JF, Rincon G, Lince D, Eggen A, Glaser L, Cam G, Van Tassel C: Analyzing LD blocks and CNV segments in cattle: novel genomic features identified using the BovineHD BeadChip. In Pub No 370-2011-002. San Diego, CA: Illumina Inc; 2011.

31. van Heerwaarden J, Hufford MB, Ross-lbarra J: Historical genomics of North American maize. Proc Natl Acad Sci U S A 2012, 109(31):12420-12425.

32. Bauer E, Falque M, Walter H, Bauland C, Camisan C, Campo L, Meyer N, Ranc N, Rincent R, Schipprack W, Altmann T, Flament P, Melchinger AE, Menz M, Moreno-Gonzalez J, Ouzunova M, Revilla P, Charcosset A, Martin OC, Schön CC: Intraspecific variation of recombination rate in maize. Genome Biol 2013, 14(9):R103

33. Lehermeier C, Krämer N, Bauer E, Bauland C, Camisan C, Campo L, Flament P, Melchinger AE, Menz M, Meyer N, Moreau L, Moreno-González J, Ouzunova M, Pausch H, Ranc N, Schipprack W, Schönleben M, Walter H, Charcosset A, Schön C-C: Usefulness of multi-parental populations of maize (Zea mays L.) for genome-based prediction. Genetics 2014, 198(1):3-16.

34. Li H, Handsaker B, Wysoker A, Fennell T, Ruan J, Homer N, Marth G, Abecasis G, Durbin R, Proc GPD: The Sequence Alignment/Map format and SAMtools. Bioinformatics 2009, 25(16):2078-2079.

35. McKenna A, Hanna M, Banks E, Sivachenko A, Cibulskis K, Kernytsky A, Garimella K, Altshuler D, Gabriel S, Daly M, DePristo MA: The Genome Analysis Toolkit: A MapReduce framework for analyzing next-generation DNA sequencing data. Genome Res 2010, 20(9):1297-1303.

36. Hufford MB, Xu X, van Heerwaarden J, Pyhajarvi T, Chia J-M, Cartwright RA, Elshire RJ, Glaubitz JC, Guill KE, Kaeppler SM, Lai J, Morrell PL, Shannon LM, Song C, Springer NM, Swanson-Wagner RA, Tiffin P, Wang J, Zhang G, Doebley J, McMullen MD, Ware D, Buckler ES, Yang S, Ross-lbarra J: Comparative population genomics of maize domestication and improvement. Nat Genet 2012, 44(7):808-811.

37. Gore MA, Chia JM, Elshire RJ, Sun Q, Ersoz ES, Hurwitz BL, Peiffer JA, McMullen MD, Grills GS, Ross-lbarra J, Ware DH, Buckler ES: A firstgeneration haplotype map of maize. Science 2009, 326(5956):1115-1117.
38. Tosser-Klopp G, Bardou P, Bouchez O, Cabau C, Crooijmans R, Dong Y, Donnadieu-Tonon C, Eggen A, Heuven HCM, Jamli S, Jiken AJ, Klopp C, Lawley CT, McEwan J, Martin P, Moreno CR, Mulsant P, Nabihoudine I, Pailhoux E, Palhiere I, Rupp R, Sarry J, Sayre BL, Tircazes A, Wang J, Wang W, Zhang WG, Consortium IGG: Design and characterization of a 52K SNP chip for goats. PLOS ONE 2014, 9(1):e86227.

39. Ramos AM, Crooijmans RPMA, Affara NA, Amaral AJ, Archibald AL, Beever JE, Bendixen C, Churcher C, Clark R, Dehais P, Hansen MS, Hedegaard J, Hu Z-L, Kerstens HH, Law AS, Megens H-J, Milan D, Nonneman DJ, Rohrer GA, Rothschild MF, Smith TPL, Schnabel RD, Van Tassell CP, Taylor JF, Wiedmann RT, Schook LB, Groenen MAM: Design of a high density SNP genotyping assay in the pig using SNPs identified and characterized by next generation sequencing technology. PLOS ONE 2009, 4(8):e6524.

40. Didion JP, Yang HN, Sheppard K, Fu CP, McMillan L, de Villena FPM, Churchill GA: Discovery of novel variants in genotyping arrays improves genotype retention and reduces ascertainment bias. BMC Genomics 2012, 13:34.

41. Labate JA, Lamkey KR, Mitchell SE, Kresovich S, Sullivan H, Smith JSC Molecular and historical aspects of corn belt dent diversity. Crop Sci 2003, 43(1):80-91.

42. Doebley J, Wendel JD, Smith JSC, Stuber CW, Goodman MM: The origin of Cornbelt maize - the isozyme evidence. Econ Bot 1988, 42(1):120-131.

43. Messmer MM, Melchinger AE, Boppenmaier J, Brunklaus-Jung E, Herrmann RG: Relationships among early European maize inbreds. 1. Genetic diversity among flint and dent lines revealed by RFLPs. Crop Sci 1992, 32(6):1301-1309.

44. Dubreuil P, Dufour P, Krejci E, Causse M, de Vienne D, Gallais A, Charcosset A: Organization of RFLP diversity among inbred lines of maize representing the most significant heterotic groups. Crop Sci 1996, 36(3):790-799.

45. Frascaroli E, Schrag TA, Melchinger AE: Genetic diversity analysis of elite European maize (Zea mays L.) inbred lines using AFLP, SSR, and SNP markers reveals ascertainment bias for a subset of SNPs. Theor Appl Genet 2013, 126(1):133-141.

46. Mikel MA: Availability and analysis of proprietary dent corn inbred lines with expired US plant variety protection. Crop Sci 2006, 46(6):2555-2560.

47. Mikel MA, Dudley JW: Evolution of North American dent corn from public to proprietary germplasm. Crop Sci 2006, 46(3):1193-1205.

48. Rebourg C, Gouesnard B, Charcosset A: Large scale molecular analysis of traditional European maize populations. Relationships with morphological variation. Heredity 2001, 86:574-587.

49. Brown WL, Anderson E: The Northern flint corns. Ann Missouri Bot Garden 1947, 34:1-28.

50. Dubreuil P, Warburton M, Chastanet M, Hoisington D, Charcosset A: More on the introduction of temperate maize into Europe: Large-scale bulk SSR genotyping and new historical elements. Maydica 2006, 51(2):281-291.

51. Rebourg C, Chastanet M, Gouesnard B, Welcker C, Dubreuil P, Charcosset A: Maize introduction into Europe: the history reviewed in the light of molecular data. Theor Appl Genet 2003, 106(5):895-903.

52. Tenaillon Ml, Charcosset A: A European perspective on maize history. C R Biol 2011, 334(3):221-228.

53. Alexander DH, Novembre J, Lange K: Fast model-based estimation of ancestry in unrelated individuals. Genome Res 2009, 19(9):1655-1664.

54. Yan JB, Shah T, Warburton ML, Buckler ES, McMullen MD, Crouch J: Genetic characterization and linkage disequilibrium estimation of a global maize collection using SNP markers. PLOS ONE 2009, 4(12):e8451.

55. Van Inghelandt D, Reif JC, Dhillon BS, Flament P, Melchinger AE: Extent and genome-wide distribution of linkage disequilibrium in commercial maize germplasm. Theor Appl Genet 2011, 123(1):11-20.

56. Nielsen R: Population genetic analysis of ascertained SNP data. Human Genomics 2004, 1(3):218-224.

57. Zila CT, Samayoa LF, Santiago R, Butrón A, Holland JB: A genome-wide association study reveals genes associated with Fusarium ear rot resistance in a maize core diversity panel. G3: Genes Genomes Genet 2013, 3(11):2095-2104.

58. Becker A, Chao D-Y, Zhang X, Salt DE, Baxter I: Bulk segregant analysis using single nucleotide polymorphism microarrays. PLOS ONE 2011, 6(1):e15993.

59. Thompson EA: Identity by descent: variation in meiosis, across genomes, and in populations. Genetics 2013, 194(2):301-326.

60. Pausch H, Aigner B, Emmerling R, Edel C, Gotz K-U, Fries R: Imputation of high-density genotypes in the Fleckvieh cattle population. Genet Sel Evol 2013, 45(1):3. 
61. Saghai-Maroof MA, Soliman KM, Jorgensen RA, Allard RW: Ribosomal DNA spacer-length polymorphisms in barley: mendelian inheritance, chromosomal location, and population dynamics. Proc Natl Acad Sci U S A 1984, 81(24):8014-8018.

62. Li H, Durbin R: Fast and accurate short read alignment with BurrowsWheeler transform. Bioinformatics 2009, 25(14):1754-1760.

63. PICARD, A set of tools (in Java) for working with next generation sequencing data in the BAM format. [http://broadinstitute.github.io/picard/]

64. Yu X, Sun S: Comparing a few SNP calling algorithms using low-coverage sequencing data. BMC Bioinformatics 2013, 14:274.

65. Cingolani P, Platts A, Wang LL, Coon M, Nguyen T, Wang L, Land SJ, Lu XY, Ruden DM: A program for annotating and predicting the effects of single nucleotide polymorphisms, SnpEff: SNPs in the genome of Drosophila melanogaster strain w(1118); iso-2; iso-3. Fly 2012, 6(2):80-92.

66. Nelson PT, Coles ND, Holland JB, Bubeck DM, Smith S, Goodman MM Molecular characterization of maize inbreds with expired US plant variety protection. Crop Sci 2008, 48(5):1673-1685.

67. Flint-Garcia SA, Thuillet AC, Yu JM, Pressoir G, Romero SM, Mitchell SE, Doebley J, Kresovich S, Goodman MM, Buckler ES: Maize association population: a high-resolution platform for quantitative trait locus dissection. Plant J 2005, 44(6):1054-1064.

68. USDA ARS National Genetic Resources Program. Germplasm Resources Information Network - (GRIN) [Online Database]. [http://www.ars-grin.gov/ cgi-bin/npgs/html/crop.pl?89]

69. Pirani A, Gao H, Bellon L, Webster T: Best practices for genotyping analysis of plant and animal genomes with Affymetrix ${ }^{\oplus}$ Axiom $^{\oplus}$ arrays. In The International Plant \& Animal Genome XXI Conference. San Diego, CA, USA: Scherago International; 2013:P0997. SNPolisher ${ }^{T M}$ is an R package available from Affymetrix Inc. and can be downloaded from the "DevNet Tools" on www.affymetrix.com.

70. R Core Team: R: A language and environment for statistical computing. 2013, http://www.R-project.org/.

71. Browning BL, Browning SR: A unified approach to genotype imputation and haplotype-phase inference for large data sets of trios and unrelated individuals. Am J Hum Genet 2009, 84(2):210-223.

72. Wimmer V, Albrecht T, Auinger HJ, Schön C-C: synbreed: a framework for the analysis of genomic prediction data using R. Bioinformatics 2012 28:2086-2087.

73. Jombart T: adegenet: a R package for the multivariate analysis of genetic markers. Bioinformatics 2008, 24(11):1403-1405.

74. Paradis E, Claude J, Strimmer K: APE: analyses of phylogenetics and evolution in R language. Bioinformatics 2004, 20:289-290.

75. Purcell S, Neale B, Todd-Brown K, Thomas L, Ferreira MAR, Bender D, Maller J, Sklar P, de Bakker PIW, Daly MJ, Sham PC: PLINK: A tool set for wholegenome association and population-based linkage analyses. Am J Hum Genet 2007, 81(3):559-575.

doi:10.1186/1471-2164-15-823

Cite this article as: Unterseer et al: A powerful tool for genome analysis in maize: development and evaluation of the high density $600 \mathrm{k}$ SNP genotyping array. BMC Genomics 2014 15:823.

\section{Submit your next manuscript to BioMed Central and take full advantage of:}

- Convenient online submission

- Thorough peer review

- No space constraints or color figure charges

- Immediate publication on acceptance

- Inclusion in PubMed, CAS, Scopus and Google Scholar

- Research which is freely available for redistribution

Submit your manuscript at www.biomedcentral.com/submit 